\title{
Postangioplasty restenosis: a practical model in the porcine carotid artery
}

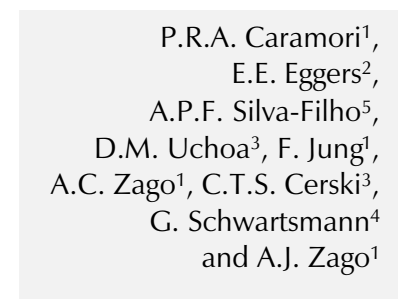

Received September 10, 1996 Accepted July 21, 1997

\author{
Serviços de ${ }^{1}$ Cardiologia, ${ }^{2}$ Cirurgia Vascular, ${ }^{3}$ Patologia and \\ ${ }^{4}$ Oncologia (South-American Office for Anticancer Drug Development), \\ Hospital de Clínicas de Porto Alegre and ${ }^{5}$ Hospital de Clínicas Veterinárias, \\ Universidade Federal do Rio Grande do Sul, Porto Alegre, RS, Brasil
}

\begin{abstract}
Correspondence
P.R.A. Caramori

Cardiovascular Clinical Research Laboratory, Mount Sinai Hospital 600 University Avenue, Suite 1609 Toronto, Ontario M5G 1X5

Canada

E-mail: caramori@outer-net.com

Research supported by CNPq and Fundo de Incentivo a Pesquisa of the Hospital de Clínicas of Porto Alegre. Dr. P.R.A. Caramori is currently a Clinical Research Fellow of the Cardiovascular Clinical Research Laboratory, University of Toronto, Canada.
\end{abstract}

\begin{abstract}
Transluminal coronary angioplasty is a routine therapeutic intervention in coronary heart disease. Despite the high rate of primary success, restenosis continues to be its major limitation. Porcine models have been considered to be the most adequate experimental models for studying restenosis. One limitation of porcine models is the need for radiological guidance and the expenses involved. The objective of the present study was to adapt an experimental model of angioplasty in the porcine carotid artery that does not require radiological equipment. Eight animals were used to develop the technique of balloon injury to the common carotid artery by dissection without radiological guidance. This technique was then employed in six other animals. Under anesthesia, the left common carotid artery was dissected and incised at the carotid sinus for insertion of an over-the-wire angioplasty balloon towards the aorta. Overstretch injury of the carotid artery was performed under direct visualization. After 30 days, the arteries were excised and pressure-fixated. Uninjured carotid arteries from 3 additional animals were used as controls. A decreased luminal area associated with intimal hyperplasia and medial reaction was observed in all injured arteries. Immunohistochemistry identified the intimal hyperplastic cells as smooth muscle cells. Computerized morphometry of the ballooned segments revealed the following mean areas: lumen $2.12 \mathrm{~mm}^{2}( \pm 1.09)$, intima $0.22 \mathrm{~mm}^{2}( \pm 0.08)$, media $3.47 \mathrm{~mm}^{2}( \pm 0.67)$, and adventitia $1.11 \mathrm{~mm}^{2}( \pm 0.34)$. Our experimental model of porcine carotid angioplasty without radiological guidance induced a vascular wall reaction and permitted the quantification of this response. This porcine model may facilitate the study of vascular injury and its response to pharmacological interventions.
\end{abstract}

Percutaneous coronary angioplasty is routinely used for the treatment of coronary heart disease. Every year, more than 300,000 angioplasties are carried out in the USA and more than 10,000 in Brazil (1). Unfortunately, some of these procedures are performed for the second or third time on the
Key words

- Arterial injury

- Intimal hyperplasia

- Restenosis

- Percutaneous transluminal coronary angioplasty same patient because recurrence of the obstruction, or restenosis, takes place in 20 to $40 \%$ of cases (2). The clinical evaluation of this phenomenon is prevented by significant limitations. First, access to human restenotic lesions for histopathological analysis is difficult. Second, clinical studies must include 
angiographic follow-up on a large number of patients, which implies risks and high operating costs. Studies on the pathophysiology of restenosis, and the assessment of strategies aimed at inhibiting this process may be performed on animal models, which allow for the selection of interventions most likely to be effective in subsequent human trials.

The major goal of these experimental models is the morphometric analysis of histological sections of the injured arteries (3). The porcine model of restenosis has shown the most consistent similarities with human restenosis (3). The histological aspect of the intimal hyperplasia and the results of experiments using the porcine common carotid artery (4), or coronary arteries (5), have been consistent with those observed in humans. One limitation of porcine models is the need for radiological guidance and the expenses that it incurs. The objective of the present study was to adapt an experimental model of balloon overstretch vascular injury of the porcine common carotid artery that dispenses with radiological equipment. This model should permit investigation of the pathophysiology of restenosis and assessment of pharmacological strategies for its prevention.

Fourteen crossbred pigs (YorkshireHampshire-Landrace) obtained from local breeders with an approximate age of 3 to 4 months and weighing 20 to $35 \mathrm{~kg}$ were used. The animals were kept in captivity and fed a commercial chow, without cholesterol or lipid supplementation. Husbandry was carried out under supervision of a veterinarian, according to international norms (6).

Adaptation of the model. To develop the technique of angioplasty of the common carotid artery by dissection, 8 animals were used. One animal was submitted to carotid angiography to assess the options for a surgical approach. No radiological guidance was used in the other procedures. Three different approaches were evaluated. Initially, an attempt was made to introduce the balloon into the common carotid artery through theexter- nal carotid artery. This access was unsuccessful because of unfavorable anatomy. Specifically, the external carotid was found to be represented by multiple small-caliber branches, frequently leaving the common carotid at a right angle. Next, the direct incision of the proximal portion of the common carotid artery close to the thoracic inlet was evaluated. This second approach had restrictions due to an insufficient surgical field. Finally, a cranial approach to the common carotid, at its bifurcation, was technically feasible and considered to be the ideal choice. All stages foreseen for the model were tested and perfected during this period, including i) sedation and anesthesia techniques, ii) histological fixing in a pressure perfusion system, and iii) morphometric analysis using a computerized system.

The angioplasty model. Once the technique was established, six additional animals were sedated with midazolam $(0.5 \mathrm{mg} /$ $\mathrm{kg}$ ), placed in dorsal decubitus and ventilated with room air and halothane to ensure adequate anesthesia. The left common carotid artery was exposed by dissection of the left anterior cervical region. Intravenous heparin (5,000 units) was given immediately before arterial access. The left common carotid artery was incised lengthwise at the carotid sinus for insertion of an over-thewire angioplasty balloon $(30 \times 8 \mathrm{~mm})$ towards the aorta. The catheter was inserted until the proximal end of the balloon was 3 $\mathrm{cm}$ beyond the arterial incision. The balloon diameter used was $8 \mathrm{~mm}$. This diameter was chosen as being approximately 1.5 times wider than the carotid artery of swine of this size $(4,7)$. Three balloon inflations were performed for $60 \mathrm{~s}$ at $6 \mathrm{~atm}$ at intervals of $60 \mathrm{~s}$. At the end of the procedure, vascular repair was done by suturing, ensuring pulsatile arterial flow. Assisted ventilation was maintained until the animals recovered. The animals received $1 \mathrm{~g}$ ampicillin, were returned to their quarters and kept under close observation. 
Tissue preparation. After 30 days, the animals were sacrificed, and the carotid arteries were removed and fixed by pressure perfusion $(100 \mathrm{mmHg}$ ) for $15 \mathrm{~min}$ in a solution of $2 \%$ glutaraldehyde and $1 \%$ paraformaldehyde in sodium phosphate buffer, $\mathrm{pH}$ 7.25 (7), followed by overnight immersion fixation. The balloon-injured portion of each artery was divided into five equally spaced annular segments, subsequently embedded in paraffin, prepared for histological sectioning, and stained using the Verhoeff and Masson trichrome techniques.

Histological analysis. Three aspects were evaluated by light microscopy, using an adapted semiquantitative graduation system, and the respective scores were recorded for each histological section: intensity of the vascular injury caused by dilatation (8), intimal reaction to injury (9), and medial reaction to injury (9) (scores 0 to ++++, Table 1). The presence of arterial thrombosis was also recorded. All measurements were independently made by two observers. In any case of discrepancy, consensus was obtained. The left carotid arteries of three animals matched for weight and age, that did not undergo angioplasty, were used for qualitative comparison. Immunoperoxidase staining for $\alpha-\gamma$ smooth muscle actin was carried out using HHF-35 monoclonal antibody (Draco, Carpinteria, CA).

Morphometric analysis. Histological images were obtained by light microscopy (Unimac, Meiji, Japan), and digitized using a video camera (CK 3800, Meiji, Japan) and an analog-digital interface (Targa plus 16, Truevision, Indianapolis, IN). The borders of the arterial wall layers were manually traced using commercially available software (Aldus PhotoStyler 2.0, Aldus Corp., Seattle, WA), operated by a single independent observer. The segments that showed the smallest luminal area in each artery were used for morphometric analysis. Planimetry was performed with the Imagescale software (Electronic Imagery, Pompano Beach, FL).
Table 1 - Semiquantitative scoring system for histological analysis of vascular injury and reaction.

Vascular injury scores were adapted from Schwartz et al. (8). Intimal and medial reaction scores were adapted from Karas et al. (9). IEL, Internal elastic lamina; EEL, external elastic lamina; SMC, smooth muscle cell.

\begin{tabular}{|c|c|c|c|}
\hline Score & Vascular injury & Intimal reaction & Medial reaction \\
\hline 0 & IEL intact & Absent & Absent \\
\hline+ & Focal IEL rupture & $\begin{array}{l}1 \text { to } 2 \mathrm{SMC} \text { layers } \\
\text { inner to IEL }\end{array}$ & $\begin{array}{l}\text { Mild increase } \\
\text { in extracellular matrix }\end{array}$ \\
\hline++ & $\begin{array}{l}\text { Medial laceration } \\
\text { restricted to inner } 1 / 2\end{array}$ & $\begin{array}{l}2 \text { to } 5 \mathrm{SMC} \text { layers } \\
\text { inner to IEL }\end{array}$ & $\begin{array}{l}\text { Moderate increase of } \\
\text { extracellular matrix, } \\
\text { restricted to inner } 1 / 3\end{array}$ \\
\hline+++ & $\begin{array}{l}\text { Medial laceration to the } \\
\text { outer } 1 / 2 \text {, with no } E E L \\
\text { compromising }\end{array}$ & $\begin{array}{l}\text { Moderate SMC } \\
\text { proliferation }\end{array}$ & $\begin{array}{l}\text { Moderate increase of } \\
\text { extracellular matrix, } \\
\text { restricted to inner } 2 / 3\end{array}$ \\
\hline++++ & Laceration of EEL & $\begin{array}{l}\text { Intense SMC } \\
\text { proliferation }\end{array}$ & $\begin{array}{l}\text { Intense and diffuse } \\
\text { increase of extracellular } \\
\text { matrix. Media disorganization }\end{array}$ \\
\hline
\end{tabular}

The arterial luminal area was obtained by direct measurement of the area delimited by the endothelium. The intimal area was calculated by subtracting the luminal area from the area delimited by the internal elastic lamina (IEL area). The medial area was determined by subtracting the IEL area from the area delimited by the external elastic lamina (EEL area). The adventitia was defined as the area between the EEL and the edge of the periadventitial adipose tissue. A millimeter scale was digitized by the same process and used for calibration. Morphometric analysis was validated by an independent laboratory using the Byltanalise KS300 system (Kontron Electronic, Eching, Germany). Inter-observer variability was less than $5 \%$.

One animal died during the procedure. Five animals and a total of 25 carotid artery segments were available for analysis. Semiquantitative microscopic analysis (Table 1) showed focal loss of continuity of the IEL in multiple points in 3 animals (score + ). In 2 animals, overstretch injury caused partial laceration of the media (score ++ ), associ- 
ated with mural thrombosis in one case and occlusive thrombosis in the other. In the latter animal, the occlusive thrombosis did not allow the identification of the internal elastic lamina in large parts of the arterial circumference, hindering definition of the intima. When compared to control arteries, all balloon-injured segments showed a marked decrease in lumen diameter, thickening of the intima and evidence of medial remodeling (Figure 1). Intimal hyperplasia was present in all balloon-injured arteries at varying intensity (score range + to ++++ ). The intima was predominantly formed by spindle-shaped cells surrounded by variable amounts of extracellular matrix. The great majority of these cells were positive for $\alpha$ and $\gamma$ smooth muscle actin by immunostaining, which identified them as smooth muscle cells. In the media, cells next to the internal elastic lamina frequently exhibited histological characteristics of activated smooth muscle cells. Some of these cells were located at sites where the internal elastic lamina was discontinued, suggesting that they might be migrating to the intima. The media showed minimal to moderate signs of reaction (score range + to +++ ). In one balloon-injured artery the media was normal. In the animals

Figure 1 - Photomicrograph of a porcine common carotid artery obtained four weeks after the balloon injury. Observe the fracture (arrow) of the internal elastic lamina associated with intimal hyperplasia. Trichrome elastic staining. Original magnification (250X).

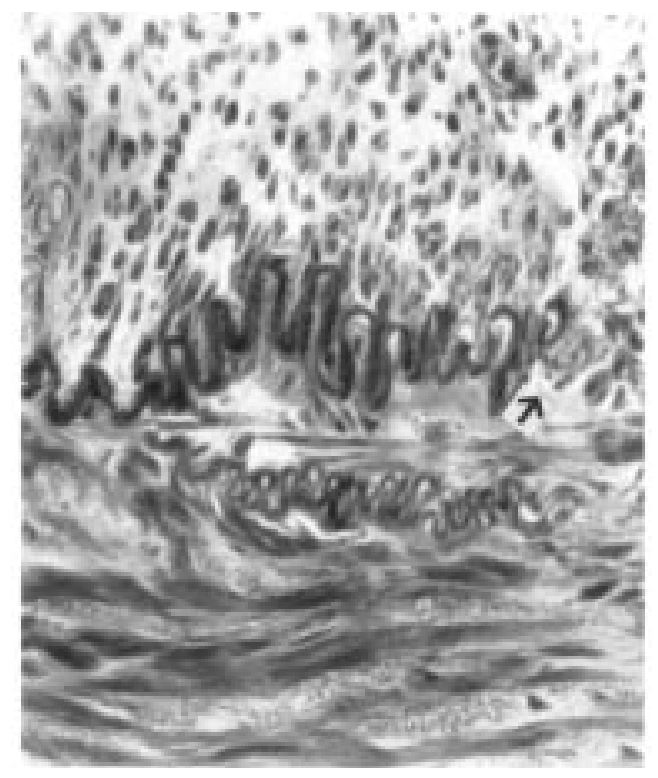

that underwent angioplasty, the computerized quantitative analysis of the digitized histological images showed a mean area of the vascular lumen of $2.12 \mathrm{~mm}^{2}( \pm 1.09)$. The average area was $0.22 \mathrm{~mm}^{2}( \pm 0.08)$ for the intima, $3.47 \mathrm{~mm}^{2}( \pm 0.67)$ for the media, and $1.11 \mathrm{~mm}^{2}( \pm 0.34)$ for the adventitia.

This experimental model of porcine carotid angioplasty without radiological guidance produced intimal hyperplasia in the majority of the animals. The diameter of the carotid arteries of crossbred pigs weighing $20-35 \mathrm{~kg}$ has been reported to be in the range of 5-6 mm. In swine of this size, other authors have used angioplasty balloons $8 \mathrm{~mm}$ in diameter for all animals $(7,10)$, despite radiological sizing of the carotid arteries. In our study we used animals of similar age and weight and a single balloon size in order to dispense with angiography. The variation in the degree of vascular injury inherent in this approach may be corrected by the injury index described. In studies evaluating the effects of interventions on the restenotic process, this correction may be particularly advisable.

One potential limitation of this model is the development of arterial thrombosis. Thrombosis is not a frequent finding in human coronary balloon angioplasty and may not play a major role in the vascular response to balloon injury. Furthermore, occlusive thrombosis does not allow further assessment of the arterial reaction to injury. In our study, arterial thrombosis was present in two cases (a mural one and an occlusive one), both of them associated with more extensive arterial injury and medial laceration (injury score $++)$. Steele et al. (7), using 8-mm balloons in pigs with an average weight of $35 \mathrm{~kg}$, reported carotid thrombotic occlusion in $11 \%$ of the animals. Muller et al. (4) used 5- to 6$\mathrm{mm}$ balloons in pigs of similar size; although carotid thrombosis was rare, some of the arteries failed to develop intimal hyperplasia. In our model, thrombosis could have been minimized by using smaller balloons. 
However, since the vascular response is proportional to the intensity of injury (8), smaller balloons might have been an ineffective stimulus for intimal hyperplasia in some animals. The administration of an antiplatelet agent could be considered in order to reduce the chances of thrombosis. However, this intervention may not be desirable in pharmacological efficacy studies.

The technical aspects developed for this experiment achieved their objectives. The pressure perfusion system of histology fixation allowed arteries to be observed under circumstances similar to their physiological condition. The score of injury showed that this method of balloon angioplasty caused controlled arterial injury. The scores of intima and media reaction allowed the identification of differences between responses from balloon-injured arteries, while morphomet- ric analysis permitted an accurate quantification of the vascular response to angioplasty.

In conclusion, we modified an experimental model of balloon injury to the porcine common carotid artery by surgical dissection. This model is feasible from a technical point of view, causes controlled vascular injury and permits quantification of the vascular reaction to injury. The lack of requirement for radiological guidance allows the study of restenosis in this porcine model at reduced cost.

\section{Acknowledgments}

We are indebted to Dr. Peter Seidelin for a critical review of the manuscript and to Dr. Carlos R.A. Caramori, Dr. João C. Prolla, Dr. Klaus Irion, and Dr. Vinícios Duval for expert assistance with the morphometric analysis.

\section{References}

1. Souza AGMR (1994). Procedimentos percutâneos de intervenção cardiovascular no Brasil em 1992 e 1993. Relatório do Registro Nacional - Centro Nacional de Intervenções Cardiovasculares. Revista Brasileira de Cardiologia Invasiva, 2: 5462.

2. Serruys $P W$, de Jaeger $P$, Kiemeneij $F$, Macaya C, Rutsch W, Heyndrickx G, Emanuelsson $\mathrm{H}$, Marco J, Legrand $\mathrm{V}$ \& Materne $P$ (1994). A comparison of balloon-expandable-stents implantation with balloon angioplasty in patients with coronary artery disease. Benestent study group. New England Journal of Medicine, 331: 489-495.

3. Muller DW, Ellis SG \& Topol EJ (1992). Experimental models of coronary artery restenosis. Journal of the American College of Cardiology, 19: 418-432.
4. Muller DWM, Topol EJ, Abrams GD, Gallagher KP \& Ellis SG (1992). Intramural methotrexate therapy for the prevention of neointimal thickening after balloon angioplasty. Journal of the American College of Cardiology, 20: 460-466.

5. Santoian ED, Schneider JE, Gravanis MB, Foegh M, Tarazona N, Cipolla GD \& King SB (1993). Angiopeptin inhibits intimal hyperplasia after angioplasty in porcine coronary arteries. Circulation, 88: 11-14.

6. Brown MJ, Pearson PT \& Tomson FN (1993). Guidelines for animal surgery in research and teaching. American Journal of Veterinary Research, 54: 1544-1559.

7. Steele PM, Chesebro JH, Stanson AW, Holmes Jr DR, Dewanjee MK, Badimon L \& Fuster V (1985). Balloon angioplasty. Natural history of the pathophysiological response to injury in a pig model. Circulation Research, 57: 105-112.
8. Schwartz RS, Huber KC, Murphy JG, Edwards WD, Camrud AR, Vliestra RE \& Holmes DR (1992). Restenosis and the proportional neointimal response to coronary artery injury: Results in a porcine model. Journal of the American College of Cardiology, 19: 267-274.

9. Karas SP, Gravanis MB, Santoian EC Robinson KA, Anderberg KA \& King SB (1992). Coronary intimal proliferation after balloon injury and stenting in swine: an animal model of restenosis. Journal of the American College of Cardiology, 20: 467-474.

10. Lam LYT, Lacoste $L$ \& Bourassa MG (1992). Cilazapril and early atherosclerotic changes after balloon injury of porcine carotid arteries. Circulation, 85: 1542-1547. 